\title{
Frameless stereotactic radiosurgery for brain metastases: a review of outcomes and prognostic scores evaluation
}

\author{
ST Mok *, Michael KM Kam, WK Tsang, Darren MC Poon, Herbert H Loong, WM Yeung, TY Yeung, \\ Jimmy Yu, Carlos KH Wong
}

- A video clip showing frameless stereotactic radiosurgery for brain metastases is available at $<w w w$ hkmj.org>

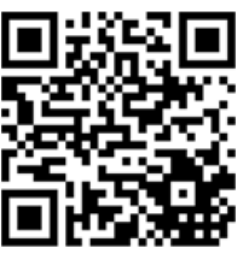

This article was published on 10 Nov 2017 at www.hkmj.org.

\section{A B S T R A C T}

Introduction: Stereotactic brain radiosurgery provides good local control in patients with limited brain metastases. A newly developed frameless system allows pain-free treatment. We reviewed the effectiveness of this frameless stereotactic brain radiosurgery and identified prognostic factors that may aid better patient selection.

Methods: Medical records of patients with brain metastases treated with linear accelerator-based frameless stereotactic brain radiosurgery between January 2010 and July 2015 in a university affiliated hospital in Hong Kong were reviewed. Outcomes including local and distant brain control rate, progression-free survival, and overall survival were analysed. Prognostic factors were identified by univariable and multivariable analyses. Association of outcomes with four common prognostic scores was performed.

Results: In this study, 64 patients with 94 lesions were treated with a median dose of 18 Gy (range, 12-22 Gy) in a single fraction. The median follow-up was 11.5 months. One-year actuarial local and distant brain control rates were $72 \%$ and $71 \%$, respectively. The median overall survival was 13.0 months. On multivariable analysis, Karnofsky performance status score ( $>50$ vs $\leq 50)$ and number of lesions (1-2 vs $\geq 3$ ) were found to associate significantly with distinct brain progression-free survival $(\mathrm{P}=0.022$, hazard ratio $=0.20$, 95\% confidence interval 0.05 0.80 and $\mathrm{P}=0.003$, hazard ratio $=0.31,95 \%$ confidence interval 0.14-0.68, respectively). Overall survival was associated significantly with Basic Score for Brain Metastases $(\mathrm{P}=0.031)$, Score Index for Radiosurgery in Brain Metastases $(\mathrm{P}=0.007)$, and Graded Prognostic Assessment $(\mathrm{P}=0.003)$. Improvement in overall survival was observed in all groups of different prognostic scores.

Conclusion: Frameless stereotactic brain radiosurgery is effective in patients with oligometastases of brain and should be increasingly considered in patients with favourable prognostic scoring.

\section{Hong Kong Med J 2017;23:599-608}

DOI: $10.12809 / \mathrm{hkmj} 166138$

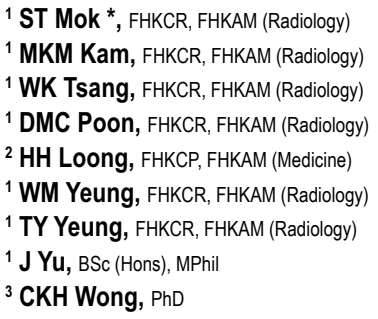

ST Mok *, FHKCR, FHKAM (Radiology)

${ }^{1}$ MKM Kam, FHKCR, FHKAM (Radiology)

${ }^{1}$ WK Tsang, FHKCR, FHKAM (Radiology)

${ }^{1}$ DMC Poon, FHKCR, FHKAM (Radiology)

${ }^{2}$ HH Loong, FHKCP, FHKAM (Medicine)

${ }^{1}$ WM Yeung, FHKCR, FHKAM (Radiology)

${ }^{1}$ TY Yeung, FHKCR, FHKAM (Radiology)

$1 \mathrm{~J}$ Yu, BSc (Hons), MPhil

${ }^{3} \mathrm{CKH}$ Wong, $\mathrm{PhD}$

\section{Department of Clinical Oncology, Prince of Wales Hospital, Shatin, Hong Kong \\ 2 Department of Clinical Oncology, The Chinese University of Hong Kong, Shatin, Hong Kong \\ ${ }^{3}$ Department of Family Medicine and Primary Care, The University of Hong Kong, Pokfulam, Hong Kong}

* Corresponding author: mst216@ha.org.hk

This paper was presented at the MASCC/ISOO meeting 2016, 23-25 June 2016, Adelaide, Australia.

New knowledge added by this study

Survival of patients with brain metastases has significantly improved over the past decade.

Frameless stereotactic brain radiosurgery is effective and has acceptable toxicities.

Implications for clinical practice or policy

- Calculation of a prognostic score can aid clinicians in the identification of patients who will benefit most from stereotactic brain radiosurgery.

\section{Introduction}

Patients with brain metastases have previously had poor survival of only 3 to 4 months with nonsurgical treatment. ${ }^{1,2}$ Substantial improvement has been achieved in recent years with the advance of systemic treatment and radiation techniques.
Stereotactic radiosurgery (SRS) was first delivered with the Cobalt-60 Gamma Knife system by Leksell in 1951. ${ }^{3}$ Today, SRS can also be delivered via the linear accelerator (LINAC) system and proton beam system. It is usually indicated in patients with oligo-brain metastases $(\leq 4)$ with a diameter of less than $4 \mathrm{~cm}^{4}$ 


\section{用於腦轉移瘤的無框架立體定位放射外科手術： 治療結果和預後評分的評估 \\ 莫小婷、甘冠明、曾偉光、潘明駿、龍浩鋒、楊會明、 楊庭恩、余啟成、黃競浩}

引言：立體定位放射外科手術能讓腦轉移瘤患者得到良好的局部控 制。新開發的無框架系統能達致無痛治療。本文回顧此技術的效用, 並找出有助選擇合適患者的預後因素。

方法：回顧香港一所大學教學醫院, 在 2010 年 1 月至2015年7月期間 腦轉移瘤患者的病歷資料, 患者均接受直線加速器結合無框架立體定 向腦放射治療。我們分析腦轉移瘤的局部和遠處控制率, 以及無進展 生存期和總生存期的結果。通過單變量和多變量分析確定預後因素, 並找出這些結果與四項常見的預後評分是否相關

結果：本研究中, 64名（94個病變）腦轉移瘤患者每次接受劑量的中 位數為 $18 \mathrm{~Gy}$ (介乎12-22 Gy) 。随訪期中位數 11.5 個月。一年統計 局部和遠處腦部控制率分別為 $72 \%$ 和 $71 \%$ 。生存期中位數 13.0 個月 多變量分析中, 發現Karnofsky行為狀態評分表 $(>50$ 比 $\leq 50)$ 和病 變數目（1-2 比 $\geq 3)$ 與腦部無進展生存顯著相關（ $\mathrm{P}=0.022$, 風險比 $=0.20 ， 95 \%$ 置信區間0.05-0.80; $\mathrm{P}=0.003$, 風險比 $=0.31,95 \%$ 置信 區間0.14-0.68）。總體生存率與腦轉移基本評分（ $\mathrm{P}=0.031 ） 、$ 放射 治療分級指數 $(P=0.007)$ 和等級預後評估標準 $(P=0.003)$ 顯著相 關。所有預後評分的不同組別的生存期均有整體改善。

結論：無框架立體定向腦放射外科手術對腦轉移瘤患者具成效，應使 用在有良好預後評分的患者中。 1-year local progression-free survival (PFS) was reported to be up to $70 \%$ to $90 \%$, and median overall survival (OS) of 6 to 12 months. ${ }^{9-15}$ The outcomes of frameless SRS have been reported only in limited series, with 1 -year local control of $79 \%$ to $95 \%{ }^{5,16-18}$

Patient selection and tailor-made management are indeed challenging. Several scoring systems have been modelled to predict survival of patients with brain metastases, including the Radiation Therapy Oncology Group Recursive Partitioning Analysis (RTOG RPA), ${ }^{19}$ Basic Score for Brain Metastases (BSBM), ${ }^{20}$ the Score Index for Radiosurgery in Brain Metastases (SIR), ${ }^{21}$ Graded Prognostic Assessment (GPA), ${ }^{22}$ and Disease-Specific Graded Prognostic Assessment (DS-GPA). ${ }^{23}$ These scoring systems were developed at a time when treatment strategies were also rapidly evolving with the availability of more accurate diagnostic imaging, better radiotherapy techniques, and more effective systemic and targeted agents. A paradigm shift to more aggressive treatment of oligo-metastasis as a result of longer cancer survivorship now requires further validation of these scoring systems.

In this study, we reviewed the outcomes of patients who underwent LINAC-based frameless SRS and identified prognostic factors that affect survival. By doing so, we hope to gain a better understanding of which patients will benefit from SRS without jeopardising their quality of life.

It is particularly advantageous for lesions in the deep brain parenchyma that are not easily accessible by surgery. A frame-based system was initially used to immobilise the patient. A frameless system was later developed to minimise patient suffering and was reported to have comparable outcomes to the framed-based system. ${ }^{5}$ Since the introduction of a frameless system, SRS or even fractionated stereotactic radiotherapy has been increasingly used to treat patients with oligo-brain metastases. Patients do not have to undergo painful frame placement. Rather, they undergo simple planning procedures over 2 consecutive days. The patient is required to return only for mould fitting and planning of computed tomography. Together with diagnostic fine-cut magnetic resonance imaging coregistration, oncologists can easily contour the target on the radiotherapy planning system. With the use of the ExacTrac system (Brainlab AG, Germany) to verify treatment position, the magnitude of error is reported to be only $0.7 \mathrm{~mm}$, and the mean deviation between frame-based and image-guided initial positioning is just $1.0 \mathrm{~mm}$ (standard deviation, 0.5 $\mathrm{mm}) .{ }^{6} \mathrm{~A}$ frameless system became one of the choices of treatment in SRS and was included in the ASTRO policy. ${ }^{7}$ The recommended dosages according to the RTOG 9005 trial are 24 Gy, 18 Gy, and 15 Gy for tumours of $\leq 20 \mathrm{~mm}, 21-30 \mathrm{~mm}$, and $31-40 \mathrm{~mm}$ in maximum diameter, respectively. ${ }^{8}$ For framed SRS,

\section{Methods}

Records of patients who underwent frameless SRS for limited brain metastases in a university-affiliated hospital between January 2010 and July 2015 were retrospectively reviewed. Patient data were extracted from paper records and the Clinical Management System of the Hospital Authority, Hong Kong by investigators in charge of the study. Data extracted included gender, age, type of primary malignancy, date of diagnosis of malignancy and brain metastases, extracranial disease status and control at treatment time, diagnostic and monitoring modalities, presence of convulsions, and steroid and anticonvulsant use before and after treatment period. Treatment details including immobilisation technique, number of lesions, dose and fractionation, and volume of lesions were reviewed from department records and the Brainlab iplan system (Brainlab AG, Germany). Prognostic scoring including RTOG RPA, BSBM, SIR, and GPA were calculated (Appendix 19-23).

Outcome parameters including local and distant brain control, PFS, and OS were generated using SPSS (Windows version 22.0; IBM Corp, Armonk [NY], US). Univariable analysis with Cox proportional hazards model was performed to generate prognostic factors for local and distinct brain PFS (defined as the time from treatment to documented local progression/distinct brain 
progression or death) and OS. For each outcome, statistically significant non-modifiable patient and disease factors in univariable analysis together with important treatment factors were included in respective multivariable analysis using Cox proportional hazards model. The enter method was used for variable selection process. Kaplan-Meier survival curve for OS was generated for different prognostic scoring groups and log rank significance was calculated. The study was approved by clinical research ethics committee of the NTEC-CUHK Cluster, Hospital Authority, Hong Kong, with patient informed consent waived.

\section{Results}

\section{Demographics}

A total of 68 patients were screened during the study period. Four patients who were treated with fractionated stereotactic radiotherapy and singlefraction SRS in the same treatment were excluded, and thus 64 patients were included. All patients were treated with frameless LINAC-based SRS with ExacTrac system verification, while contouring and dosimetry with the Brainlab iplan system. Dose administered was based on tumour diameter: $22 \mathrm{~Gy}$ to lesions of $\leq 2 \mathrm{~cm}, 18$ Gy to lesions of $2.1-3.0 \mathrm{~cm}$, and 15 Gy to lesions of $3.1-4.0 \mathrm{~cm}$. A $1.5-\mathrm{mm}$ margin was allowed from gross tumour volume to planning target volume. Deviation of dose prescription from departmental protocol was permitted at the individual physician's discretion.

Among the 64 patients, there were 40 men and 24 women. The median age at the time of treatment was 58 years (range, 22-95 years). The median Eastern Cooperative Oncology Group performance status was 1 (range, 0-3), and Karnofsky Performance Status (KPS) score was 80 (range, 40-100). Primary disease included carcinoma of breast $(n=7)$, lung $(n=45)$, gastrointestinal $(n=2)$, renal cell $(n=6)$, thyroid $(n=1)$, osteosarcoma $(n=1)$, germ cell $(n=1)$, and epithelioid haemangioendothelioma $(n=1)$. Further details of demographics are summarised in Table 1.

\section{Treatment}

A total of 94 lesions were treated with a dose of 12 Gy to 22 Gy according to size (12 Gy, n=6; 15 Gy, $\mathrm{n}=12 ; 16$ Gy, $\mathrm{n}=2 ; 18 \mathrm{~Gy}, \mathrm{n}=48 ; 20 \mathrm{~Gy}, \mathrm{n}=14 ; 22 \mathrm{~Gy}$, $\mathrm{n}=12$ ). The median dose was $18 \mathrm{~Gy}$. The median size of lesion treated was $19 \mathrm{~mm}$ (range, 3-43 mm).

\section{Outcomes}

The median follow-up time was 11.5 (range, 0.456.4) months. One-year actuarial local control rate was $72 \%$ (95\% confidence interval [CI], 57\%-83\%) and distant control rate was $71 \%$ (95\% CI, 56\%-82\%). The median local PFS was 11.2 (95\% CI, 8.4-11.2) months. The median distinct brain PFS was 10.8 (95\% CI, 8.4-13.1) months. The median OS was 13.0 (95\% CI, 10.6-11.3) months.

\section{Toxicities}

Four $(6.3 \%)$ patients had acute toxicities, mainly brain oedema, and one patient had a seizure for 3 days after treatment. Eight (12.5\%) patients had delayed seizure after a median time of 10.5 months. One patient had radionecrosis confirmed pathologically after surgical resection. There were 43 (67.2\%) patients who were prescribed steroid before treatment, and eight (12.5\%) patients became steroid dependent until their demise. Steroid prescription was not found to affect OS significantly. Nonetheless among the steroid group, becoming steroid dependent was associated with poorer prognosis, with a median OS in the steroid-dependent group of 0.92 months versus 13.6 months in the non-steroiddependent group $(\mathrm{P}<0.005, \log$ rank; Appendix 2, Fig a). The worse survival of steroid-dependent patients was independent of volume of brain metastases.

\section{Prognostic patient and disease factors}

Potential prognostic factors of survival including patient factors such as gender, age, and performance status; and disease factors such as primary cancer, presence of extracranial disease, pre-existing convulsion, number of brain lesions, and size and volume of the largest lesion were examined with reference to decision for SRS treatment by univariable analysis using Cox proportional hazards model. It was found that OS was associated significantly with age ( $\leq 70$ vs $>70$ years; $\mathrm{P}=0.011)$ and KPS score ( $>50$ vs $\leq 50 ; \mathrm{P}=0.008$ ). Local $\mathrm{PFS}$ was associated significantly with age ( $\leq 70$ vs $>70$ years; $\mathrm{P}=0.043$ ) and KPS score ( $>50$ vs $\leq 50 ; \mathrm{P}=0.021$ ). Distinct brain PFS was associated significantly with age ( $\leq 70$ vs $>70$ years; $\mathrm{P}=0.02$ ), presence of extracranial disease (presence vs absence; $\mathrm{P}=0.038$ ), KPS score ( $>50$ vs $\leq 50 ; \mathrm{P}=0.009)$, and number of brain lesions $(<1-2$ vs $\geq 3 ; \mathrm{P}=0.016$ ). Results of univariable analysis are summarised in Table 2.

\section{Treatment factors}

\section{Dose relationship}

Dose relationship for each lesion was analysed separately. Lesions prescribed $>18$ Gy had statistically significant superior time to progression (radiologically documented local progression) than those given $\leq 18 \mathrm{~Gy}$, with a 1-year local control rate of $88 \%$ vs $60 \%$ (Appendix 2, Fig b). Some patients had more than one lesion treated with different doses. Nonetheless after taking into account the highest dose given in the same patient, dose did not affect local PFS or OS significantly (Table 2); dose was not analysed in distinct brain PFS as it should not affect 
TABLE I. Demographics and treatment details

\begin{tabular}{|c|c|}
\hline & $\begin{array}{l}\text { No. }(\%) \text { of } \\
\text { patients }\end{array}$ \\
\hline \multicolumn{2}{|l|}{ Gender } \\
\hline Male & $40(62.5)$ \\
\hline Female & $24(37.5)$ \\
\hline \multicolumn{2}{|l|}{ Age-group (years) } \\
\hline $18-30$ & $2(3.1)$ \\
\hline $31-40$ & $6(9.4)$ \\
\hline $41-50$ & $11(17.2)$ \\
\hline $51-60$ & $18(28.1)$ \\
\hline $61-70$ & $18(28.1)$ \\
\hline $71-80$ & $8(12.5)$ \\
\hline$>80$ & $1(1.6)$ \\
\hline \multicolumn{2}{|l|}{ Primary malignancy (subtype) } \\
\hline Breast & $7(10.9)$ \\
\hline Luminal A & 1 \\
\hline Luminal B & 4 \\
\hline Her2 & 2 \\
\hline Basal-like & 0 \\
\hline Lung & $45(70.3)$ \\
\hline NSCLC/adenoCa NOS & 16 \\
\hline NSCLC/adenoCa unknown mutation & 1 \\
\hline NSCLC/adenoCa EGFR mutant* & 17 \\
\hline ALK+ & 3 \\
\hline $\mathrm{SqCC}$ & 5 \\
\hline SCLC & 2 \\
\hline Unknown & 1 \\
\hline Gastrointestinal & $2(3.1)$ \\
\hline Renal cell carcinoma & $6(9.4)$ \\
\hline Thyroid (papillary) & $1(1.6)$ \\
\hline Osteosarcoma & $1(1.6)$ \\
\hline Germ cell & $1(1.6)$ \\
\hline Epithelioid haemangioendothelioma & $1(1.6)$ \\
\hline \multicolumn{2}{|l|}{ ECOG performance status } \\
\hline 0 & $5(7.8)$ \\
\hline 1 & $41(64.1)$ \\
\hline 2 & $15(23.4)$ \\
\hline 3 & $3(4.7)$ \\
\hline \multicolumn{2}{|l|}{ Extracranial disease control } \\
\hline Uncontrolled & $27(42.2)$ \\
\hline Controlled & $22(34.4)$ \\
\hline Complete remission & $15(23.4)$ \\
\hline \multicolumn{2}{|c|}{ 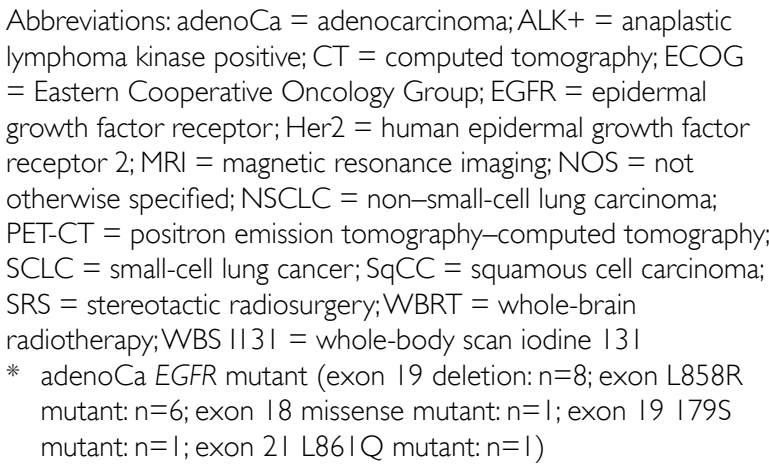 } \\
\hline
\end{tabular}

TABLE I. (cont'd)

\begin{tabular}{cc}
\hline & $\begin{array}{c}\text { No. (\%) of } \\
\text { patients }\end{array}$ \\
\hline Presence of extracranial metastases & \\
Yes & $42(65.6)$ \\
No & $22(34.4)$
\end{tabular}

No. of brain lesions

$\begin{array}{lc}1 & 37(57.8) \\ 2 & 16(25.0) \\ 3 & 8(12.5) \\ 4 & 1(1.6) \\ \geq 5 & 2(3.1) \\ \text { Imaging modality } & \\ \text { At treatment } & \\ \text { CT only } & 5(7.8) \\ \text { CT and MRI } & 59(92.2) \\ \text { Monitoring } & \\ \text { CT } & 35(54.7) \\ \text { PET-CT } & 3(4.7) \\ \text { MRI } & 16(25.0) \\ \text { WBS I131 } & 1(1.6) \\ \text { Nil } & 9(14.1)\end{array}$

Pre-existing convulsion

$\begin{array}{ll}\text { Yes } & 11(17.2) \\ \text { No } & 53(82.8)\end{array}$

Breakthrough convulsion

Yes

No

$56(87.5)$

Steroid use during SRS

Yes $43(67.2)$

No $\quad 19(29.7)$

Unknown 2 (3.1)

Steroid dependent afterwards

$\begin{array}{lc}\text { Yes } & 8(12.5) \\ \text { No } & 54(84.4) \\ \text { Unknown } & 2(3.1)\end{array}$

WBRT

WBRT >3 months before $10(15.6)$

Concomitant WBRT 25 (39.1)

Salvage WBRT $9(14.1)$

Lifelong WBRT $43(67.2)$

Progressive disease after SRS

Local

10 (15.6)

Distinct 23 (35.9)

Salvage treatment on disease progression

\begin{tabular}{ll} 
WBRT & $9(14.1)$ \\
SRS & $1(1.6)$ \\
Surgery & $1(1.6)$ \\
\hline
\end{tabular}


(a)

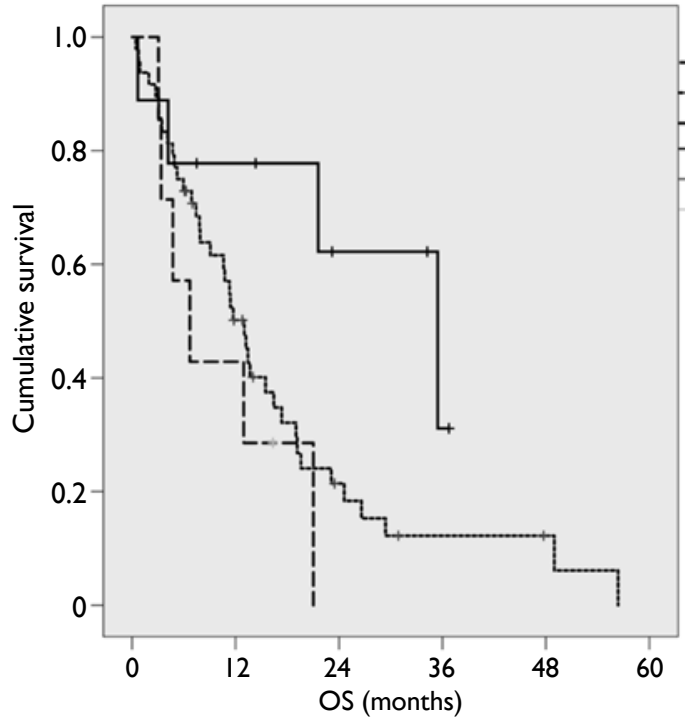

(c)

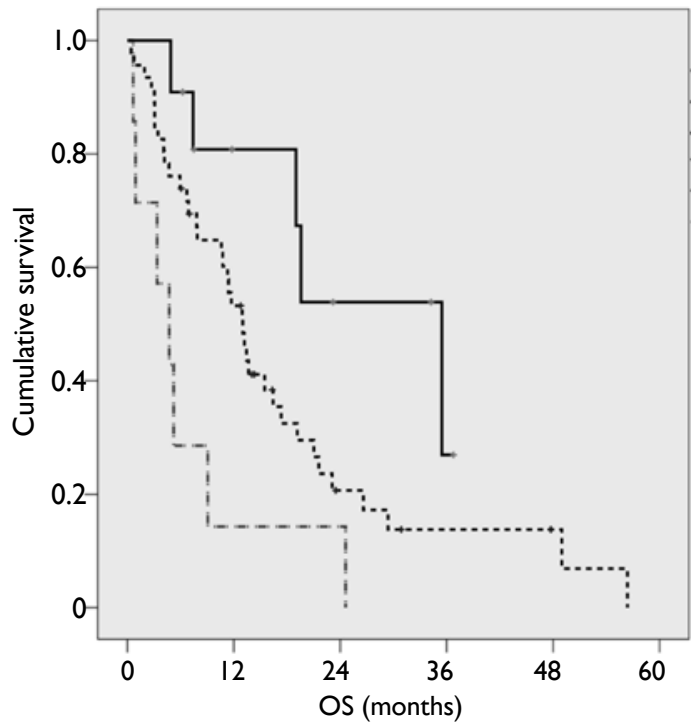

(b)
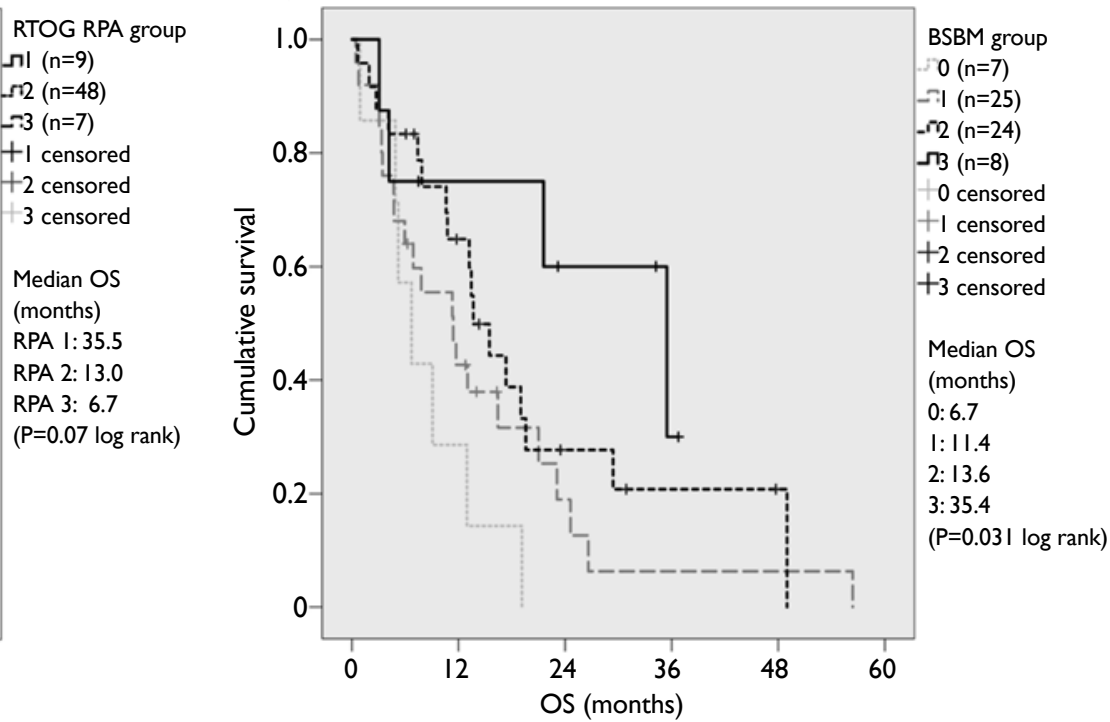

(d)

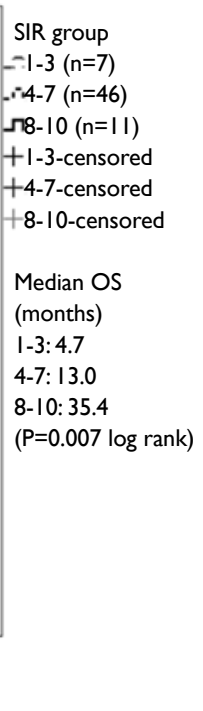

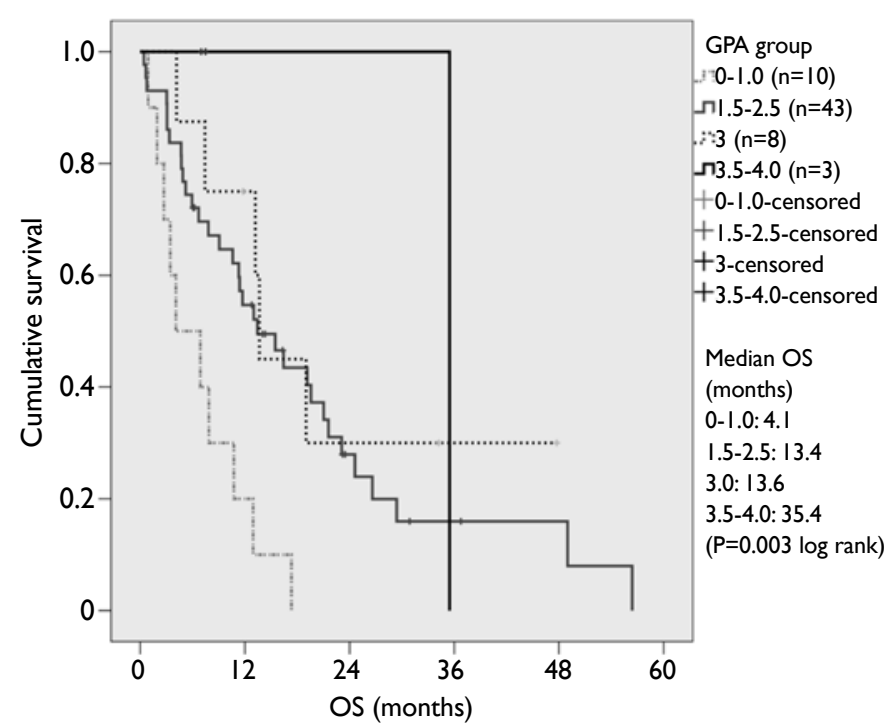

FIG. Overall survival (OS) according to prognostic score grouping: (a) RTOG RPA, (b) BSBM, (c) SIR, and (d) GPA

Abbreviations: BSBM = Basic Score for Brain Metastases; GPA = Graded Prognostic Assessment ; RTOG RPA = Radiation Therapy Oncology Group Recursive Partitioning Analysis; SIR = Score Index for Radiosurgery in Brain Metastases

distant brain progression.

\section{Effect of whole-brain radiotherapy}

With particular reference to the effect of whole-brain radiotherapy (WBRT), it was found that concomitant WBRT (within 3 months of treatment with SRS) did not have a statistically significant impact on OS, local PFS, or distant brain PFS (Table 2).

\section{Multivariable analysis} hazards model and taking patient, disease, and EGFR mutation (exon 19 deletion or exon 21 L858R treatment factors into account identified that mutation) was present in 14. Three other patients statistically significant factors associated with distinct brain PFS were KPS score ( $>50$ vs $\leq 50$; $\mathrm{P}=0.022$, hazard ratio $[\mathrm{HR}]=0.20,95 \% \mathrm{CI}=0.05-0.80)$ and number of brain lesions (1-2 vs $\geq 3 ; \mathrm{P}=0.003$, $\mathrm{HR}=0.31,95 \% \mathrm{CI}=0.14-0.68$ ) [Table 3].

\section{Primary lung cancer}

Of note, a large number of patients in the group had primary lung cancer $(n=45)$, most of which were non-small-cell lung cancer (NSCLC) $[\mathrm{n}=42]$.
Among NSCLC patients, a sensitive activating 
TABLE 2. Univariable analysis by categorical variables

\begin{tabular}{|c|c|c|c|c|c|c|c|}
\hline \multirow[t]{2}{*}{ Variable } & \multirow{2}{*}{$\begin{array}{l}\text { No. }(\%) \text { of } \\
\text { patients }\end{array}$} & \multicolumn{2}{|c|}{ Local PFS } & \multicolumn{2}{|c|}{ Overall survival } & \multicolumn{2}{|c|}{ Distinct brain PFS } \\
\hline & & HR (95\% Cl) & $P$ value & HR (95\% Cl) & $P$ value & HR (95\% Cl) & $P$ value \\
\hline \multicolumn{8}{|l|}{ Patient factors } \\
\hline \multicolumn{8}{|l|}{ Gender } \\
\hline Male & $40(62.5)$ & $1.21(0.69-2.14)$ & 0.502 & $1.249(0.69-2.27)$ & 0.47 & $1.02(0.58-1.77)$ & 0.95 \\
\hline Female & $24(37.5)$ & & & & & & \\
\hline \multicolumn{8}{|l|}{ Age (years) } \\
\hline$\leq 70$ & $56(87.5)$ & $0.45(0.21-0.97)$ & 0.043 & $0.61(0.40-0.89)$ & 0.011 & $0.40(0.19-0.86)$ & 0.02 \\
\hline$>70$ & $8(12.5)$ & & & & & & \\
\hline \multicolumn{8}{|l|}{ KPS score } \\
\hline$>50$ & 61 (95.3) & $0.49(0.27-0.90)$ & 0.021 & $0.43(0.23-0.80)$ & 0.008 & $0.44(0.24-0.81)$ & 0.009 \\
\hline$\leq 50$ & $3(4.7)$ & & & & & & \\
\hline \multicolumn{8}{|l|}{ KPS score } \\
\hline$\geq 80$ & 46 (71.9) & $0.74(0.41-1.34)$ & 0.32 & $0.63(0.34-1.17)$ & 0.149 & $0.78(0.43-1.44)$ & 0.43 \\
\hline$<80$ & $18(28.1)$ & & & & & & \\
\hline \multicolumn{8}{|l|}{ Disease factors } \\
\hline \multicolumn{8}{|l|}{ Primary cancer } \\
\hline Lung & $45(70.3)$ & $1.12(0.62-2.02)$ & 0.71 & $1.01(0.74-1.38)$ & 0.95 & $1.18(0.65-2.12)$ & 0.59 \\
\hline Non-lung & $19(29.7)$ & & & & & & \\
\hline \multicolumn{8}{|l|}{ Presence of extracranial disease } \\
\hline $\begin{array}{l}\text { Presence (disease under control/ } \\
\text { not under control) }\end{array}$ & $49(76.6)$ & $1.25(0.90-1.73)$ & 0.19 & $1.41(2.98-2.01)$ & 0.06 & $2.06(1.04-4.08)$ & 0.038 \\
\hline $\begin{array}{l}\text { Complete remission (no disease } \\
\text { except brain) }\end{array}$ & $15(23.4)$ & & & & & & \\
\hline \multicolumn{8}{|l|}{ No. of brain lesions } \\
\hline $1-2$ & $53(82.8)$ & $0.76(0.54-1.08)$ & 0.127 & $0.73(0.51-1.04)$ & 0.08 & $0.66(0.46-0.93)$ & 0.016 \\
\hline$\geq 3$ & $11(17.2)$ & & & & & & \\
\hline \multicolumn{8}{|l|}{ Pre-existing convolution } \\
\hline Presence & $11(17.2)$ & $1.12(0.79-1.59)$ & 0.52 & $1.06(0.73-1.52)$ & 0.78 & $1.04(0.73-1.49)$ & 0.83 \\
\hline Absence & $53(82.8)$ & & & & & & \\
\hline \multicolumn{8}{|l|}{ Size of the largest lesion (mm) } \\
\hline$<20$ & $35(54.7)$ & $0.83(0.63-1.09)$ & 0.18 & $0.65(0.36-1.16)$ & 0.15 & $0.86(0.65-1.13)$ & 0.29 \\
\hline$\geq 20$ & $29(45.3)$ & & & & & & \\
\hline \multicolumn{8}{|l|}{ Volume of the largest lesion $(\mathrm{mL})$} \\
\hline$<10$ & $33(51.6)$ & $1.28(0.71-2.12)$ & 0.46 & $1.38(0.77-2.46)$ & 0.27 & $1.26(0.72-2.18)$ & 0.42 \\
\hline$\geq 10$ & $31(48.4)$ & & & & & & \\
\hline \multicolumn{8}{|l|}{ Treatment factors } \\
\hline \multicolumn{8}{|l|}{ Dose (Gy) } \\
\hline$>18$ (any lesion in the same patient) & $20(31.3)$ & $0.78(0.57-1.07)$ & 0.12 & $0.84(0.61-1.15)$ & 0.15 & N/A & N/A \\
\hline$\leq 18$ & $44(68.8)$ & & & & & & \\
\hline \multicolumn{8}{|l|}{ Concomitant WBRT } \\
\hline Yes & $25(39.1)$ & $0.85(0.64-1.13)$ & 0.27 & $0.90(0.67-1.22)$ & 0.51 & $0.89(0.67-1.18)$ & 0.43 \\
\hline No & $39(60.9)$ & & & & & & \\
\hline \multicolumn{8}{|l|}{ Steroid use* } \\
\hline Yes & $43(67.2)$ & $1.24(0.91-1.70)$ & 0.17 & $1.36(0.96-1.91)$ & 0.081 & $1.16(0.85-1.58)$ & 0.36 \\
\hline No & $19(29.7)$ & & & & & & \\
\hline
\end{tabular}

Abbreviations: $\mathrm{Cl}$ = confidence interval; $\mathrm{HR}$ = hazard ratio; KPS = Karnofsky performance status; N/A = not applicable; PFS = progression-free survival; WBRT = whole-brain radiotherapy

* Data were missing for two cases 
TABLE 3. Multivariable analysis by categorical variables with Cox regression model

\begin{tabular}{|c|c|c|c|c|c|c|c|c|c|}
\hline \multirow[t]{2}{*}{ Variable } & \multicolumn{3}{|c|}{ Local PFS } & \multicolumn{3}{|c|}{ Overall survival } & \multicolumn{3}{|c|}{ Distinct brain PFS } \\
\hline & $\begin{array}{l}\text { Median } \\
\text { survival } \\
\text { (months) }\end{array}$ & HR (95\% Cl) & $P$ value & $\begin{array}{c}\text { Median } \\
\text { survival } \\
\text { (months) }\end{array}$ & HR (95\% Cl) & $P$ value & $\begin{array}{l}\text { Median } \\
\text { survival } \\
\text { (months) }\end{array}$ & HR (95\% Cl) & $P$ value \\
\hline \multicolumn{10}{|l|}{ Age (years) } \\
\hline$\leq 70$ & 11.3 & $0.84(0.54-1.32)$ & 0.45 & 13.4 & $0.76(0.48-1.21)$ & 0.25 & 11.3 & $0.42(0.16-1.06)$ & 0.067 \\
\hline$>70$ & 3.3 & & & 3.4 & & & 2.5 & & \\
\hline \multicolumn{10}{|l|}{ KPS score } \\
\hline$>50$ & 11.3 & $0.61(0.31-1.18)$ & 0.14 & 13.4 & $0.27(0.07-1.00)$ & 0.068 & 11.3 & $0.20(0.05-0.80)$ & 0.022 \\
\hline$\leq 50$ & 4.7 & & & 4.7 & & & 2.4 & & \\
\hline $\begin{array}{l}\text { Presence of extracranial } \\
\text { disease }\end{array}$ & & N/A & & & N/A & & & & \\
\hline $\begin{array}{l}\text { Presence (uncontrolled/ } \\
\text { controlled) }\end{array}$ & & & & & & & 9.0 & $1.10(0.55-2.22)$ & 0.78 \\
\hline Absence & & & & & & & 17.0 & & \\
\hline No. of brain lesions & & N/A & & & N/A & & & & \\
\hline $1-2$ & & & & & & & 11.3 & $0.31(0.14-0.68)$ & 0.003 \\
\hline$\geq 3$ & & & & & & & 5.8 & & \\
\hline Dose (Gy) & & & & & & & & N/A & \\
\hline$>18$ & 11.4 & $0.77(0.55-1.06)$ & 0.11 & 19.1 & $0.87(0.62-1.21)$ & 0.40 & & & \\
\hline$\leq 18$ & 9.8 & & & 13.0 & & & & & \\
\hline \multicolumn{10}{|l|}{ Concomitant WBRT } \\
\hline Yes & 12.9 & $0.81(0.58-1.12)$ & 0.20 & 13.6 & $0.90(0.64-1.26)$ & 0.53 & 12.9 & $0.56(0.45-1.66)$ & 0.66 \\
\hline No & 7.4 & & & 10.7 & & & 7.4 & & \\
\hline \multicolumn{10}{|l|}{ Steroid use } \\
\hline Yes & 9.0 & $1.25(0.89-1.76)$ & 0.19 & 11.4 & $1.32(0.91-1.91)$ & 0.15 & 10.4 & $1.29(0.63-2.66)$ & 0.48 \\
\hline No & 14.0 & & & 21.5 & & & 11.3 & & \\
\hline
\end{tabular}

Abbreviations: $\mathrm{Cl}$ = confidence interval; $\mathrm{HR}$ = hazard ratio; $\mathrm{KPS}$ = Karnofsky performance status; N/A = not applicable; PFS = progression-free survival; WBRT = whole-brain radiotherapy

carried a less common mutation: exon 21 861Q lines of EGFR-TKI after SRS treatment.

$(\mathrm{n}=1)$, exon 18 missense $(\mathrm{n}=1)$, and exon $18179 \mathrm{~S}$

$(\mathrm{n}=1)$. Patients with an exon 19 deletion or exon 21

L858R mutation had superior OS compared with the non-mutational group $(\mathrm{P}=0.019, \mathrm{HR}=0.281$, 95\% $\mathrm{CI}=0.097-0.814)$ but there was no statistically significant difference in local or distant brain control. Among the 14 patients with sensitive activating EGFR mutation, three patients who were diagnosed with brain metastases received WBRT before SRS treatment, and six patients were given SRS together with WBRT. Again, concomitant WBRT was not shown to affect local/distinct brain PFS or OS. For epidermal growth factor receptor (EGFR)-tyrosine kinase inhibitor (TKI) treatment, 12 of 14 patients had lifelong EGFR-TKI treatment, with a median survival of 19.5 months; one with exon 19 deletion and one with exon 18 missense deletion did not have EGFR-TKI treatment. There were seven patients who were prescribed EGFR-TKI before SRS treatment (range of duration, 5.7-21.4 months), and eight patients who were started on or continued on more

\section{Association with available prognostic scoring}

Overall survival was significantly associated with BSBM ( $\mathrm{P}=0.031$, log-rank), SIR ( $\mathrm{P}=0.007$, log-rank), and GPA ( $\mathrm{P}=0.003$, log-rank) [Fig]. A comparison of median survival of the current study with the other original studies is shown in Table $4 .{ }^{19-22}$ Of note, DS-GPA was not analysed due to the small number of patients with breast, gastrointestinal, and renal cell primaries. The calculation of GPA and DS-GPA of lung primary was the same.

\section{Discussion}

Brain metastasis has previously been considered an end-of-life event. With the development of new systemic therapies that are effective in both extracranial and intracranial diseases, together with a better understanding from clinical trials of the advantages of SRS, oncologists are more willing to offer SRS to patients with limited brain metastases. 
TABLE 4. Comparison of median overall survival in the present study according to prognostic scoring group with that of original studies $^{19-22}$

\begin{tabular}{ccc}
\hline & \multicolumn{2}{c}{ Median overall survival time (months) } \\
\cline { 2 - 3 } & Original study & Present study \\
\hline RTOG GPA, ${ }^{19}{ }^{1997}$ & & \\
I & 7.7 & 35.5 \\
III & 4.5 & 13.0 \\
BSBM, ${ }^{20}$ 2004 & 2.3 & 6.7 \\
\hline 3 & & \\
\hline 2 & 7.0 & 35.5 \\
\hline 1 & 5.1 & 13.7 \\
\hline 0 & 3.4 & 11.4 \\
\hline SIR, ${ }^{21} 2000$ & 2.2 & 6.7 \\
$8-10$ & & \\
\hline $4-7$ & 8.8 & 35.5 \\
\hline $1-3$ & 6.0 & 13.0 \\
\hline GPA, ${ }^{22} 2008$ & 2.1 & 4.7 \\
\hline $3.5-4.0$ & & \\
\hline 3 & 11.0 & 35.5 \\
\hline $1.5-2.5$ & 6.9 & 13.7 \\
\hline $0-1.0$ & 3.8 & 13.4 \\
\hline
\end{tabular}

Abbreviations: BSBM = Basic Score for Brain Metastases; GPA = Graded Prognostic Assessment; RTOG RPA = Radiation Therapy Oncology Group Recursive Partitioning Analysis; SIR = Score Index for Radiosurgery in Brain Metastases

At the other extreme, studies have compared the efficacy of WBRT with supportive care in patients with advanced brain metastases. The latest news from the QUARTZ trial, conducted by the UK Medical Research Council Group, presented at the American Society of Clinical Oncology Meeting in $2015^{24}$ (full paper awaited) was striking for oncologists. They randomly allocated 538 NSCLC patients with brain metastases that were not amenable to surgery or SRS to either optimal supportive care (OSC) plus WBRT (20 Gy/5 fractions) or OSC alone. There was no significant difference in survival between the OSC+WBRT group and OSC-alone group, with the median survival being 65 and 57 days, respectively. Quality of life was also assessed in this study. The difference between the mean quality-adjusted life-years was -1.9 days only (OSC+WBRT 43.3 vs OSC-alone 41.4 days) and did not meet the initial defined criteria of significance. These data revealed that we are encountering a group of patients with very heterogeneous tumour behaviour and thus personalised treatment is required.

This retrospective study included patients who underwent frameless SRS during January 2010 to July 2015, after commencement of frameless SRS treatment in our centre. Limitations of this study including small number of patients and information bias are inevitable. Nonetheless, the outcomes of patients with brain metastases who underwent frameless SRS in our centre are compatible with those from other large clinical trials that used frame-based systems in terms of control rate, median OS and PFS, and toxicities. Approximately $13 \%$ of patients had a complication of steroid dependence that may have been due to treatment or natural disease progression. Steroid dependence was associated with poor survival, independent of volume of tumour. Prolonged use of steroid has been associated with decreased immunity that may underlie superimposed infection. Therefore, tailing down of steroid dose as early as possible in accordance with patient symptoms is strongly recommended.

This study revealed that OS was significantly associated with previously identified prognostic scoring group such as BSBM, SIR, and GPA. Among the three, BSBM and GPA are more convenient to use as only three or four factors are considered respectively, and the information should be easily available in a clinic (including age, KPS, control of primary cancer, presence of extracranial metastases, and number of brain metastases). Data relating to volume of the largest brain lesion included in SIR may not always be available as the reporting radiologist may only report lesion diameter. In terms of patient selection, for patients with GPA of $0-1.0$, the median OS was 4.1 months in our study compared with 2.6 months in the original study, and similar to that of patients given WBRT alone. It may be more appropriate to prescribe WBRT alone or best supportive care for this group of patients in lieu of SRS. An important observation from the result of our study is that survival of patients was significantly improved compared with a previous cohort (Table 4). This reflects a significant improvement in systemic treatment over the last decade. Thus, the use of high technology radiation techniques such as SRS is increasingly considered by radiation oncologists to achieve the best outcomes.

Another important aim of this study was to identify prognostic factors of survival in order to avoid futile treatment in those patients who will have a poor outcome despite SRS. Due to the small number of patients in this study, we were not able to identify patients with superior survival among different primaries, similar to DS-GPA. It is of note that a large number of patients in our study had primary lung cancer. In the NSCLC subgroup, patients with an activating EGFR mutation had significantly better survival than those without mutation, and the majority of this group had EGFRTKI lifelong. Of note, EGFR-TKI has been shown in various studies to have PFS and survival benefit 
in patients with EGFR-activating mutation. ${ }^{25-32}$ In a recent retrospective multi-institutional study with more than 300 patients, outcomes of patients with EGFR-activating mutation were analysed following treatment with upfront SRS followed by EGFR-TKI, upfront WBRT followed by EGFR-TKI, and upfront EGFR-TKI. ${ }^{33}$ Patients in the upfront SRS and upfront WBRT group had significantly superior OS and intracranial PFS compared with those with upfront EGFR-TKI. ${ }^{33}$ Therefore, in patients with oligo-brain metastases harbouring an EGFR-activating mutation, SRS followed by EGFR-TKI should be considered a standard treatment, and WBRT reserved until there is frank brain disease progression to conserve cognitive function. In addition, SRS combined with efficacious systemic treatment with good brain penetration while omitting WBRT should also be considered in other primaries, although further studies are awaited to validate the benefit.

The beneficial effect of WBRT in addition to SRS is controversial. Recent evidence shows it improves local control but not survival. ${ }^{34,35}$ Nonetheless, in view of toxicity of somnolence, malaise and cognitive impairment with WBRT, many clinicians may prefer delaying WBRT until there is frank disease progression after SRS. In a recent meta-analysis, the benefit of additional WBRT was not observed in patients who were 50 years old or younger in terms of survival or distant brain control. ${ }^{36}$ Initial omission of WBRT in this young age-group had no adverse effect on distant brain relapse rate. We were unable to replicate improvement in brain control with WBRT or demonstrate an interaction of age with benefit of concomitant WBRT, possibly due to the small size and retrospective nature of our current study. Number of brain metastases was identified as a significant prognostic factor of brain PFS. Patients with three or more brain metastases had worse PFS than those with one or two brain metastases (5.8 months vs 11.3 months). Again due to the small number of patients, we were unable to demonstrate whether concomitant WBRT could improve brain PFS in patients with three or more brain metastases. Further prospective studies are warranted to verify whether concomitant WBRT should be considered in patients with a higher disease load or age over 50 years.

Frameless SRS for oligo-brain metastases is painless and well tolerated, and should be increasingly considered in patients with good prognostic scores. Its combination with effective systemic treatment has significantly improved survival over the past decade. Nonetheless it is important to individualise treatment for patients with brain metastases according to their inherited prognostic risk factors. High precision treatment with SRS with or without WBRT should be offered to patients with oligobrain metastases with good prognostic scores and favourable primary histology. For patients with EGFR-activating mutation, SRS followed by EGFRTKI is a superior choice of treatment. Based on the latest evidence, it may be advisable to give SRS alone and reserve WBRT as salvage for patients with limited brain metastases who are 50 years or younger. Further, WBRT alone can be offered to patients with multiple symptomatic brain metastases and unfavourable prognostic scores. Best supportive care with dexamethasone alone may be considered for patients with very poor performance status.

\section{Conclusion}

Frameless SRS is effective and safe for patients with oligo-metastases of brain. Identification of patients with brain metastases who would benefit from SRS is important. Current available prognostic scoring systems provide a good estimation of survival. Frameless SRS should be increasingly considered in patients with favourable prognostic scores.

\section{Appendices}

Additional material related to this article can be found on the HKMJ website. Please go to <http:// www.hkmj.org >, and search for the article.

\section{Acknowledgements}

I would like to thank Dr SF Leung and Dr Kennis Ngar of Department of Clinical Oncology, Prince of Wales Hospital, Hong Kong for their professional opinion and support in this study.

\section{Declaration}

All authors have disclosed no conflicts of interest.

\section{References}

1. Tsao MN, Lloyd NS, Wong RK, et al. Radiotherapeutic management of brain metastases: a systematic review and meta-analysis. Cancer Treat Rev 2005;31:256-73.

2. Nieder C, Spanne O, Mehta MP, Grosu AL, Geinitz H. Presentation, patterns of care, and survival in patients with brain metastases: what has changed in the last 20 years? Cancer 2011;117:2505-12.

3. Leksell L. The stereotaxic method and radiosurgery of the brain. Acta Chir Scand 1951;102:316-9.

4. Lo SS. Stereotactic radiosurgery. Available from: http:// emedicine.medscape.com/article/1423298-overview\#a2. Accessed 19 Mar 2015.

5. Minniti G, Scaringi C, Clarke E, Valeriani M, Osti M, Enrici RM. Frameless linac-based stereotactic radiosurgery (SRS) for brain metastases: analysis of patient repositioning using a mask fixation system and clinical outcomes. Radiat Oncol 2011;6:158

6. Ramakrishna N, Rosca F, Friesen S, Tezcanli E, Zygmanszki P, Hacker F. A clinical comparison of patient setup and intra-fraction motion using frame-based radiosurgery versus a frameless image-guided radiosurgery system for 
intracranial lesions. Radiother Oncol 2010;95:109-15.

7. ASTRO Policies for Stereotactic radiosurgery (SRS). Available from: https://www.astro.org/uploadedFiles/ Main_Site/Practice_Management/Reimbursement/ ASTROSRSModelPolicy.pdf. Accessed 2014.

8. Shaw E, Scott C, Souhami L, et al. Single dose radiosurgical treatment of recurrent previously irradiated primary brain tumors and brain metastases: final report of RTOG protocol 90-05. Int J Radiat Oncol Biol Phys 2000;47:291-8.

9. Sneed PK, Lamborn KR, Forstner JM, et al. Radiosurgery for brain metastases: is whole brain radiotherapy necessary? Int J Radiat Oncol Biol Phys 1999;43:549-58.

10. Pirzkall A, Debus J, Lohr F, et al. Radiosurgery alone or in combination with whole-brain radiotherapy for brain metastases. J Clin Oncol 1998;16:3563-9.

11. Andrews DW, Scott CB, Sperduto PW, et al. Whole brain radiation therapy with or without stereotactic radiosurgery boost for patients with one to three brain metastases: Phase III results of the RTOG 9508 randomised trial. Lancet 2004;363:1665-72.

12. Cho KH, Hall WA, Gerbi BJ, Higgins PD, Bohen M, Clark HB. Patient selection criteria for the treatment of brain metastases with stereotactic radiosurgery. J Neurooncol 1998;40:73-86.

13. Varlotto JM, Flickinger JC, Niranjan A, Bhatnagar AK, Kondziolka D, Lunsford LD. Analysis of tumor control and toxicity in patients who have survived at least one year after radiosurgery for brain metastases. Int J Radiat Oncol Biol Phys 2003;57:452-64.

14. Bhatnagar AK, Flickinger JC, Kondziolka D, Lunsford LD. Stereotactic radiosurgery for four or more intracranial metastases. Int J Radiat Oncol Biol Phys 2006;64:898-903.

15. Nieder C, Grosu AL, Gaspar LE. Stereotactic radiosurgery (SRS) for brain metastases: a systematic review. Radiat Oncol 2014;9:155.

16. Pham NL, Reddy PV, Murphy JD, et al. Frameless, realtime, surface imaging-guided radiosurgery: update on clinical outcomes for brain metastases. Transl Cancer Res 2014;3:351-7.

17. Breneman JC, Steinmetz R, Smith A, Lamba M, Warnick RE. Frameless image-guided intracranial stereotactic radiosurgery: clinical outcomes for brain metastases. Int J Radiat Oncol Biol Phys 2009;74:702-6.

18. Muacevic A, Kufeld M, Wowra B, Kreth FW, Tonn JC. Feasibility, safety, and outcome of frameless image-guided robotic radiosurgery for brain metastases. J Neurooncol 2010;97:267-74.

19. Gaspar L, Scott C, Rotman M, et al. Recursive partitioning analysis (RPA) of prognostic factors in three Radiation Therapy Oncology Group (RTOG) brain metastases trials. Int J Radiat Oncol Biol Phys 1997;37:745-51.

20. Lorenzoni J, Devriendt D, Massager N, et al. Radiosurgery for treatment of brain metastases: estimation of patient eligibility using three stratification systems. Int J Radiat Oncol Biol Phys 2004;60:218-24.

21. Weltman E, Salvajoli JV, Brandt RA, et al. Radiosurgery for brain metastases: a score index for predicting prognosis. Int J Radiat Oncol Biol Phys 2000;46:1155-61.

22. Sperduto PW, Berkey B, Gaspar LE, Mehta M, Curran W. A new prognostic index and comparison to three other indices for patients with brain metastases: an analysis of 1,960 patients in the RTOG database. Int J Radiat Oncol Biol Phys 2008;70:510-4.

23. Sperduto PW, Kased N, Roberge D, et al. Summary report on the graded prognostic assessment: an accurate and facile diagnosis-specific tool to estimate survival for patients with brain metastases. J Clin Oncol 2012;30:419-25.

24. Mulvenna PM, Nankivell MG, Barton R, et al. Whole brain radiotherapy for brain metastases from non-small lung cancer: Quality of life (QoL) and overall survival (OS) results from the UK Medical Research Council QUARTZ randomised clinical trial (ISRCTN 3826061). J Clin Oncol 2015;33(Suppl);abstract8005.

25. Mok TS, Wu YL, Thongprasert S, et al. Gefitinib or carboplatin-paclitaxel in pulmonary adenocarcinoma. N Engl J Med 2009;361:947-57.

26. Han JY, Park K, Kim SW, et al. First-SIGNAL: first-line single-agent iressa versus gemcitabine and cisplatin trial in never-smokers with adenocarcinoma of the lung. J Clin Oncol 2012;30:1122-8.

27. Mitsudomi T, Morita S, Yatabe Y, et al. Gefitinib versus cisplatin plus docetaxel in patients with non-small-cell lung cancer harbouring mutations of the epidermal growth factor receptor (WJTOG3405): an open label, randomised phase 3 trial. Lancet Oncol 2010;11:121-8.

28. Maemondo M, Inoue A, Kobayashi K, et al. Gefitinib or chemotherapy for non-small-cell lung cancer with mutated EGFR. N Engl J Med 2010;362:2380-8.

29. Jänne PA, Wang X, Socinski MA, et al. Randomized phase II trial of erlotinib alone or with carboplatin and paclitaxel in patients who were never or light former smokers with advanced lung adenocarcinoma: CALGB 30406 trial. J Clin Oncol 2012;30:2063-9.

30. Rosell R, Carcereny E, Gervais R, et al. Erlotinib versus standard chemotherapy as first-line treatment for European patients with advanced EGFR mutation-positive nonsmall-cell lung cancer (EURTAC): a multicentre, open-label, randomised phase 3 trial. Lancet Oncol 2012;13:239-46.

31. Zhou C, Wu YL, Chen G, et al. Erlotinib versus chemotherapy as first-line treatment for patients with advanced EGFR mutation-positive non-small-cell lung cancer (OPTIMAL, CTONG-0802): a multicentre, open-label, randomised, phase 3 study. Lancet Oncol 2011;12:735-42.

32. Yang JC, Wu YL, Schuler M, et al. Afatinib versus cisplatinbased chemotherapy for EGFR mutation-positive lung adenocarcinoma (LUX-Lung 3 and LUX-Lung 6): analysis of overall survival data from two randomised, phase 3 trials. Lancet Oncol 2015;16:141-51.

33. Magnuson WJ, Lester-Coll NH, Wu AJ, et al. Management of brain metastases in tyrosine kinase inhibitor-naïve epidermal growth factor receptor-mutant non-small-cell lung cancer: a retrospective multi-institutional analysis. J Clin Oncol 2017;35:1070-7.

34. Mehta MP, Tsao MN, Whelan TJ, et al. The American Society for Therapeutic Radiology and Oncology (ASTRO) evidence-based review of the role of radiosurgery for brain metastases. Int J Radiat Oncol Biol Phys 2005;63:37-46.

35. Patil CG, Pricola K, Sarmiento JM, Garg SK, Bryant A, Black KL. Whole brain radiation therapy (WBRT) alone versus WBRT and radiosurgery for the treatment of brain metastases. Cochrane Database Syst Rev 2012;(9):CD006121.

36. Sahgal A, Aoyama H, Kocher M, et al. Phase 3 trials of stereotactic radiosurgery with or without whole-brain radiation therapy for 1 to 4 brain metastases: individual patient data meta-analysis. Int J Radiat Oncol Biol Phys 2015;91:710-7. 